\title{
Thermal modifications of mesons, and restoration of broken symmetries from spatial correlation functions with HISQ action
}

\author{
Yu Maezawa* \\ Yukawa Institute for Theoretical Physics, Kyoto University, Kyoto 606-8502, Japan \\ E-mail: maezawa@yukawa.kyoto-u.ac.jp
}

\section{Frithjof Karsch}

Physics Department, Brookhaven National Laboratory, Upton, NY 11973, USA

Fakultät für Physik, Universität Bielefeld, D-33615 Bielefeld, Germany

\section{Swagato Mukherjee}

Physics Department, Brookhaven National Laboratory, Upton, NY 11973, USA

\section{Peter Petreczky}

Physics Department, Brookhaven National Laboratory, Upton, NY 11973, USA

\begin{abstract}
We investigate modifications of meson states, and restorations of broken symmetries at finite temperature utilizing spatial correlation functions computed using lattice QCD. The lattice QCD computations are carried out using the Highly Improved Staggered Quark action with dynamical physical strange quark and nearly-physical up/down quarks. We compute spatial correlation functions of meson states consisting of up/down, strange and charm quarks in the temperature range of $0.7 \leq T / T_{c} \leq 2.6, T_{c}$ being the transition temperature. We extract the screening masses from the exponential decays of the spatial correlators. Continuum extrapolated results reveal that, irrespective of the flavor content of the meson states, with rising temperatures the screening masses in the negative parity channel monotonically increase, whereas those in the positive parity channel first decrease and then increase. From the degeneracies of the chiral partners of the light meson states we find that the restoration of the chiral symmetry occurs at $T_{c}$, but the axial $U_{A}(1)$ symmetry remains manifestly broken till $T \sim 1.5 T_{c}$.
\end{abstract}

The 33rd International Symposium on Lattice Field Theory

14 - 18 July 2015

Kobe International Conference Center, Kobe, Japan*

\footnotetext{
* Speaker.
} 


\section{Introduction}

Thermal fluctuations in QCD matter cause a lot of notable properties which play an important role to understand the extremely high temperature phase, i.e. the Quark-Gluon Plasma (QGP), expected to be created in the relativistic heavy-ion collision experiments at RHIC and LHC. Interquark correlations also change in QGP medium, which gives rise to modifications of hadronic excitation. In a constituent quark picture the interaction between quark and antiquark in a meson state becomes weakened due to the screening effect in the medium, as well as the quarks lose the constituent masses due to the restoration of broken chiral symmetry. Those effects may cause several modifications to the meson states which have been expected to be observed in the heavy-ion collision experiments, e.g. in-medium modifications and dissolution of charmonium were proposed as a signal of the transition to QGP phase [1] and the existence of heavy-light mesons above the transition temperature was also suggested to cause the large energy loss and flow of heavy quarks [2]. Therefore theoretical understanding of meson states at high temperature is important to reveal the nature of QGP and heavy-ion collision experiments.

The lattice simulations is the most effective method to investigate non-perturbative aspects of QCD in the 1st principle approach. Information on a meson state can be extracted from a long distance behavior to temporal direction of the current-current correlation functions. This becomes difficult at finite temperature since the lattice simulations are performed in Euclidean space-time and the temporal extent is limited by the inverse of temperature $1 / T$. To expose properties of the in-medium mesons from the limited temporal data several approaches have been proposed, such as the maximum entropy method, the variational technique etc. [3].

On the other hand, the spatial correlation functions is free from such a limitation. The spatial correlation function $G(z, T)$ can also access to information of the thermal modifications of mesonic excitation via the spectral function $\sigma(T)$ as [4]:

$$
G(z, T)=\int_{0}^{\infty} \frac{2 d \omega}{\omega} \int_{-\infty}^{\infty} d p_{z} e^{i p_{z} z} \sigma\left(\omega, p_{z}, T\right) .
$$

The spectral function defined as the Fourier transform of a real time meson correlation function is the basic quantity providing information of the in-medium properties of mesons. Although the relation of the spectral functions to the spatial correlation function is more complicated than that to the temporal one, the right-hand-side of Eq. (1.1) does not include the temperature dependence except for $\sigma(T)$ thus the ratio $G(z, T) / G(z, 0)$ becomes a direct indicator of thermal modifications of the spectral functions [5].

The spatial correlation functions decay exponentially at large distance, characterized by the decay constant known as the screening mass $M$, i.e. $G(z, T) \stackrel{z \rightarrow \infty}{\longrightarrow} \exp (-M(T) z)$. At limiting cases the screening mass shows well known behavior: At enough low temperature where the mesonic bound state exists as the ground state of the correlators, the spectral function becomes $\sigma \sim \delta\left(\omega^{2}-p_{z}^{2}-m_{0}^{2}\right)$ with $m_{0}$ being the meson pole mass at $T=0$ and thus $M(T) \sim m_{0}$. On the other hand, at high temperature limit which corresponds to perturbative or non-interacting limit the spatial correlation functions becomes a free quark-antiquark pair then $M_{\text {free }}(T)=\sqrt{(\pi T)^{2}+m_{q 1}^{2}}+\sqrt{(\pi T)^{2}+m_{q 2}^{2}}$ where $m_{q 1,2}$ are the bare mass of the valence quark and antiquark and $\pi T$ is the lowest Matsubara frequency coming from the anti-periodic boundary condition to the temporal direction. 
Moreover the spatial correlation functions are utilizable to investigate the restorations of broken symmetries at finite temperature. The chiral symmetry mixes the parity partner states and its broken in the vacuum causes mass splittings of those states, e.g. splitting between pseudo-scalar and scalar channels, and vector and axial-vector channels. Furthermore the scalar partners are mixed under the axial $U_{A}(1)$ symmetry [6]. Those broken symmetries are (effectively) restored at high temperature and then the separated partner states will degenerate. Thus the degeneracies of those parity partners provide information of the restoration patterns of the broken symmetries. It is known that such restoration patterns have played an important role in studies of the phase structure of QCD [7].

\section{Lattice simulations}

The spatial correlation functions and screening masses have been studies in lattice simulations with quenched approximation [8,9] as well as full-QCD approach with the staggered actions $[10,11,12,13,14]$ and the Wilson actions [15]. By using the Highly Improved Staggered Quarks (HISQ) action the screening masses have been calculated for the meson states consisting of the strange $(s)$ and charm $(c)$ quarks [5] and, in this report, we develop those to including the light, up/down $(u / d)$ quarks. Although the HISQ action is known to have smaller taste symmetry violations than the other staggered actions currently used in studies of the QCD thermodynamics [16], contributions of the violations become serious in the light flavors. Thus the continuum extrapolation is indispensable to suppress the non-trivial contributions of the taste violating see-quarks and to provide quantitative results of the screening masses for the light flavor sectors.

We compute the spatial correlation functions on gauge configurations generated with $2+1$ flavor QCD with the HISQ action [16, 17]. The strange quark mass $m_{s}$ is fixed on the physical value and the light quark mass are set to be $m_{u / d}=m_{s} / 20$, corresponding to $m_{\pi} \simeq 160 \mathrm{MeV}$ and $m_{K} \simeq 504$ $\mathrm{MeV}$ at zero temperature. The charm quarks are treated only as the valence ones and the mass is adjusted to reproduce a spin-averaged charmonium mass, $\left(m_{\eta_{c}}+3 m_{J / \psi}\right) / 4$ at zero temperature. The lattice sizes, $N_{s}^{3} \times N_{\tau}$, are chosen as $N_{\tau}=8,10$ and 12 with fixing $N_{s} / N_{\tau}=4$, which enables the continuum extrapolations. The lattice couplings are $\beta=6.050-7.030,6.488-7.150$ and $6.740-$ 7.825 for $N_{\tau}=8,10$ and 12 , respectively, which cover the temperature range of $0.71 \leq T / T_{c} \leq 2.60$ with $T_{c}$ being the chiral crossover temperature in the continuum limit $T_{c}=154 \pm 9 \mathrm{MeV}$ [16].

A quark contains four valence tastes in the staggered formulations and meson operators are defined by using the Dirac Gamma matrices which generate spin and taste structures [18]: $J_{H}=$ $\bar{q}\left(\Gamma^{D} \times \Gamma^{F}\right) q$ and we focus only on local meson operators: $\Gamma^{D}=\Gamma^{F}=\Gamma$. With staggered quark fields $\chi(\mathbf{x})$ at $\mathbf{x}=(x, y, z, \tau)$ the meson operators is written as $J_{H}(\mathbf{x})=\tilde{\phi}(\mathbf{x}) \bar{\chi}(\mathbf{x}) \chi(\mathbf{x})$, where $\tilde{\phi}(\mathbf{x})$ is a phase factor depending on the choice of $\Gamma$. We consider four channels for the phase factor, pseudo-scalar, scalar, vector and axial-vector, and extract the ground states of those channels from large distance behavior of the correlation functions, corresponding to $J^{P}=0^{-}, 0^{+}, 1^{-}$and $1^{+}$, respectively.

In this report we calculate only the quark-line connected part of the meson correlators and neglect the disconnected part which does not contribute to the flavor non-singlet $u \bar{d}$ mesons and $u \bar{s}$ and $u \bar{c}$ mesons. In the case of charmonium $(c \bar{c})$ the contribution of the disconnected part is small, see e.g. Ref.[19]. For $s \bar{s}$ case the disconnected part causes mixing with the flavor singlet $u \bar{d}$ sector. Fortunately for the $J^{P}=1^{ \pm}$channels because of the ideal mixing, $1^{-}$and $1^{+}$states obtained by 
the connected part in the lattice simulations are well identified with physical $\phi$ and $f_{1}(1420)$ states, respectively, at zero temperature [5]. At high temperature the contribution of the disconnected part will be suppressed due to the screening effect.

Since two different parity states couple in the staggered correlators, the large distance behavior of the meson correlators can be described by

$$
G(z, T)=A_{N O}^{2}\left(e^{-M_{-} z}+e^{-M_{-}\left(N_{z}-z\right)}\right)-(-1)^{z} A_{O}^{2}\left(e^{-M_{+} z}+e^{-M_{+}\left(N_{z}-z\right)}\right),
$$

where the first (second) term on the right-hand-side characterizes a non-oscillating (oscillating) part describing the negative (positive) parity state. Corresponding physical states we consider at zero temperature is $\left(0^{-}, 0^{+}, 1^{-}, 1^{+}\right)=\left(\pi,-, \rho, a_{1}\right)$ for the flavor non-singlet $u \bar{d}$ mesons, $\left(K, K_{0}^{*}, K^{*}, K_{1}\right)$ for $u \bar{s},\left(D, D_{0}^{*}, D^{*}, D_{1}\right)$ for $u \bar{c},\left(\eta_{s \bar{s}},-, \phi, f_{1}(1420)\right)$ for $s \bar{s},\left(D_{s}, D_{s 0}^{*}, D_{s}^{*}, D_{s 1}\right)$ for $s \bar{c}$ and $\left(\eta_{c}, \chi_{c 0}, J / \psi, \chi_{c 1}\right)$ for $c \bar{c}$, where $\eta_{s \bar{s}}$ is an unphysical state with the mass $M_{\eta_{s \bar{s}}}=\sqrt{2 M_{K}^{2}-M_{\pi}^{2}}$. We avoid identifications of $0^{+}$states of $u \bar{d}$ and $s \bar{s}$ because the former is not stable state at zero temperature due to decay to $2 \pi$ states on the lattice and the latter does not have corresponding physical state due to neglecting the disconnected part discussed above. The screening masses of those states are used as the indicators of the restorations of broken symmetries.

\section{Results}

We have calculated the spatial correlation functions of mesons consisting of the light $(u / d)$, strange $(s)$ and charm $(c)$ quarks and extracted the screening masses by fitting those at large distance with Eq. (2.1) using Bayesian fits [20]. In this report we show only results of the screening masses and those of the spatial correlation functions will be summarized in [21].

Figure 1 shows the temperature dependence of the screening masses for all meson states we consider in $\mathrm{GeV}$ unit, where the circle, diamond, triangle and anti-triangle plots indicate $0^{-}, 0^{+}$, $1^{-}$and $1^{+}$states, respectively, and colored-open, black-open and colored-filled symbols indicate results of $N_{\tau}=8,10$, and 12 , respectively. The yellow band expresses the crossover transition temperature $T_{c}$ and the lines in the left-hand-side show results of corresponding spectra on the lattice at $T=0$. The colored curves near the data express results of the continuum extrapolations with uncertainties shown by shadows. We neglect the data of the charmed mesons at $\beta<6.4$ on $N_{\tau}=8$ where the charm quark mass becomes larger than the cutoff scale.

We find that there is no significant deviations between different $N_{\tau}$ s which implies the HISQ action suppresses well the effects of the taste symmetry violation in the case of the screening mass, too. At low temperature the screening masses show almost equivalent values to the corresponding spectra at $T=0$, and show temperature dependence with $T$ increasing. The temperature dependence is found to be universal for all meson states: The negative parity states $\left(0^{-}\right.$and $\left.1^{-}\right)$increase monotonically with temperature, whereas the positive parity states $\left(0^{+}\right.$and $\left.1^{+}\right)$first decrease and then increase at some temperatures. The turning temperatures of the positive parity states seem to be flavor dependent: The increasing occurs at higher temperature for heavier mesons. Then the parity partner states close each other with temperature increasing.

The degeneracies of parity partner states can be seen at some temperatures. In particular, for $u \bar{d}$ the vector partners, $1^{-}$and $1^{+}$, degenerate at $T \sim T_{c}$ which suggests that the restoration of broken 
chiral symmetry occurs at the temperature. This is proper because the transition temperature have been determined from the chiral condensate and its susceptibility [16]. On the other hand, the mass splitting between the scalar partners, $0^{-}$and $0^{+}$, is explicit at $T \sim T_{c}$ and both states degenerate at $T \sim 1.5 T_{c}$ which implies that breaking of the $U_{A}(1)$ symmetry is significant at $T=T_{c}$ since the splitting above $T_{c}$ is caused by the $U_{A}(1)$ breaking. The results are consistent with the lattice calculations of low-lying eigen modes of the Dirac matrix on the HISQ configurations [22]. In the other flavor channels the mass splittings of the parity partners are explicit at $T=T_{c}$ and the degeneracies appear above the transition temperature. Both degeneracies seem to appear at the same temperature, e.g. at $T \sim 1.5 T_{c}$ for $u \bar{s}$ and $u \bar{c}$ mesons, $T \sim 1.7 T_{c}$ for $s \bar{s}$, etc.

At high temperature the correlation functions are described by the free theory where the quarkantiquark pair can get to be a bound state no longer. The free screening masses, $M_{\text {free }}(T)$ with the quark masses in $\overline{\mathrm{MS}}$ scheme at $2 \mathrm{GeV}$, are also shown in Fig. 1 by the dashed-black lines, and one finds that the lattice screening masses become closer at higher temperature, in particular for $u \bar{d}$, $u \bar{s}$ and $s \bar{s}$ mesons. Furthermore linear-like behavior of the screening masses at high temperature implies that the system is almost described by the free quark-antiquark pair. Such behavior is shown at similar temperature for the light and open-flavor mesons, i.e. $T \sim 1.1 T_{c}$ for $u \bar{d}$ and $T \sim 1.2-1.3 T_{c}$ for $u \bar{s}$ and $u \bar{c}$.
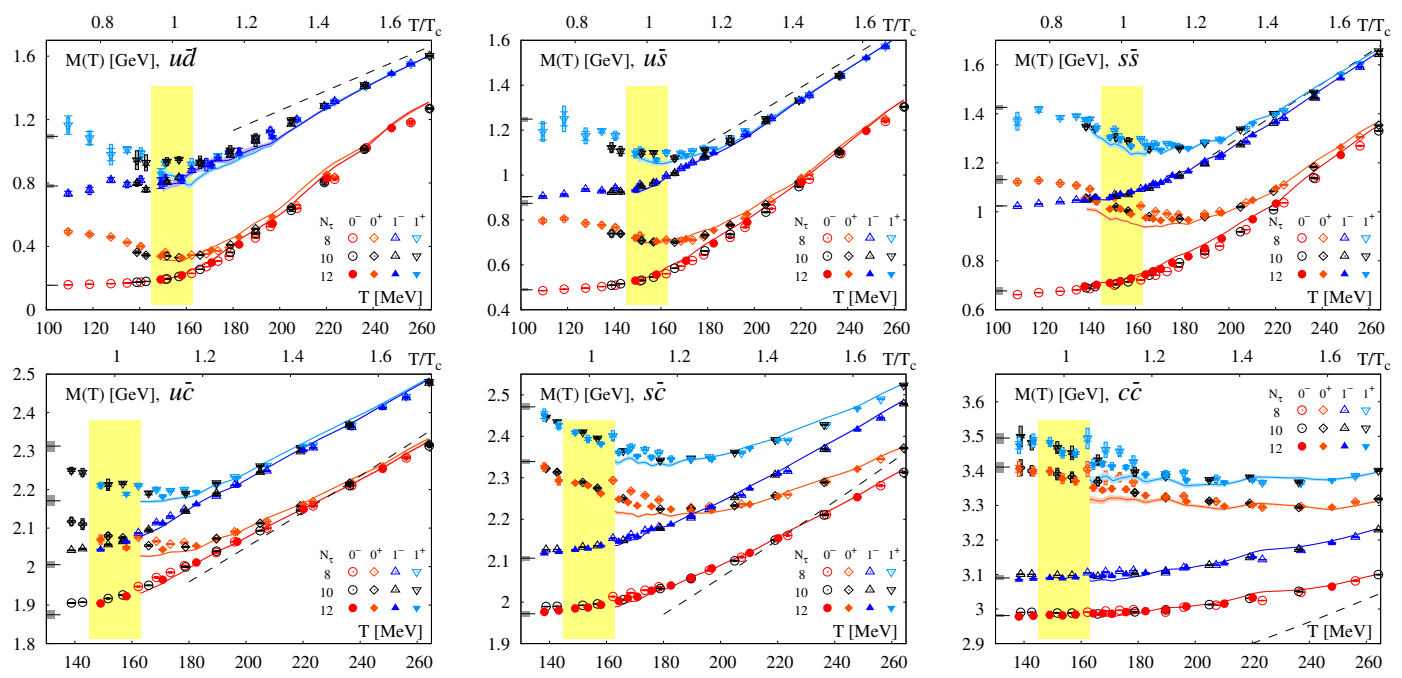

Figure 1: Screening masses for all meson states we calculate as function of temperature. The dashed lines indicate the free screening masses $M_{\text {free }}(T)$, definition is in the text.

To investigate thermal modifications of meson states in more detail we introduce the difference between the screening mass and the corresponding pole mass $m_{0}$ at $T=0: \Delta M(T)=M(T)-m_{0}$. The difference provides an information of the change in the biding energy, as long as the spatial correlation function can describe a meson bound state at low and intermediate temperature. We show $\Delta M(T)$ in Fig. 2 for $0^{-}$and $0^{+}$states in the left panel and $1^{-}$and $1^{+}$states in the right panel for $u \bar{d}, u \bar{s}, u \bar{c}$ and $s \bar{c}$ mesons, where we omit the results of $u \bar{d} 0^{+}$states since this is unstable at $T=0$. For simplicity we show only the results of $N_{\tau}=12$ and the continuum extrapolations. Similar results for $s \bar{s}, s \bar{c}$ and $c \bar{c}$ mesons have been shown and discussed in Ref. [5].

We find that $\Delta M(T)$ becomes positive (negative) values for the negative (positive) parity states, 
corresponding to the direct behavior of the screening masses shown in Fig. 1. In the negative parity $\left(0^{-}\right.$and $\left.1^{-}\right)$states the thermal modification pattern of $u \bar{d}, u \bar{s}$ and $u \bar{c}$ mesons is very similar below $T_{c}$, whereas a clear flavor dependence appears $T \gtrsim 1.1 T_{c}$ for $0^{-}$and $\gtrsim 1.3 T_{c}$ for $1^{-}$, where lighter mesons suffer larger modifications. On the other hand, the temperature dependence of $s \bar{c}$ mesons is not on such a modification pattern. In particular, $0^{-}$states have the lightest mass in the flavor sectors and have dominant contributions to the equation of states of the matter. Therefore such a property of the flavor dependence can probably be measured in the susceptibilities with respect to the quark chemical potential $[23,24]$. On the other hand, explicit thermal modifications and flavor dependence appear in the positive parity states below and above $T_{c}$.
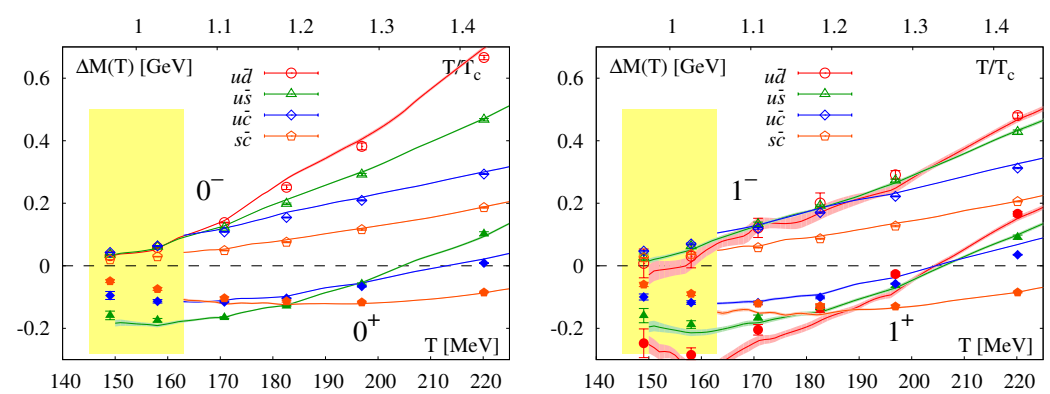

Figure 2: Difference between the screening masses at $T>0$ and the pole masses at $T=0$ for $u \bar{d}, u \bar{s}$ and $u \bar{c}$ mesons in the scalar (left) vector (right) channels.

\section{Summary}

We investigated the spatial correlation functions and screening masses at finite temperature for the meson states consisting of up/down, strange and charm quarks. Lattice QCD calculations were carried out in $2+1$ flavors of HISQ action. From the long-distance behaviors of the spatial correlators we extracted the screening masses of the ground states in the scalar $\left(J^{P}=0^{-}\right.$and $\left.0^{+}\right)$channels and vector $\left(1^{-}\right.$and $\left.1^{+}\right)$channels. We found that the screening masses of the negative parity states monotonically increase with increasing temperature. On that other hand, the screening masses for the positive parity states first decrease and then increase as temperature rises. These patterns are independent of the flavor content of the meson states. The restoration of chiral symmetry, measured through the degeneracy of the screening masses of the parity partners in the vector channel, occurs at $T \sim T_{c}$. The breaking of axial $U_{A}(1)$ symmetry breaking were quantified by measuring the non-degeneracy of the screening masses of the parity partners in the scalar channel, and the $U_{A}(1)$ symmetry was found to remain manifestly broken till $T \sim 1.5 T_{c}$.

\section{Acknowledgments:}

Numerical calculations were carried out on the USQCD Clusters at Jefferson Laboratory and the GPU Clusters at Bielefeld University. This material is based upon work supported by the U.S. Department of Energy, Office of Science, Office of Nuclear Physics, under the Contract No. DESC0012704.

\section{References}

[1] T. Matsui and H. Satz, J/4 Suppression by Quark-Gluon Plasma Formation. Phys. Lett., B178, 416, 1986. 
[2] R. Sharma et al., Light-cone wave function approach to open heavy flavor dynamics in QCD matter. Phys. Rev., C80:054902, 2009.

[3] A. Mocsy et al. Quarkonia in the Quark Gluon Plasma. Int. J. Mod. Phys., A28:1340012, 2013.

[4] F. Karsch and E. Laermann, Thermodynamics and in medium hadron properties from lattice QCD. In *Hwa, R.C. (ed.) et al.: Quark gluon plasma* 1-59, 2003.

[5] A. Bazavov et al., In-medium modifications of open and hidden strange-charm mesons from spatial correlation functions. Phys. Rev., D91(5):054503, 2015.

[6] E. Shuryak, Which chiral symmetry is restored in hot QCD? Comments Nucl. Part. Phys., 21(4):235-248, 1994.

[7] R. Pisarski and F. Wilczek, Remarks on the Chiral Phase Transition in Chromodynamics. Phys. Rev., D29:338-341, 1984.

[8] C. Detar and J. Kogut, Measuring the Hadronic Spectrum of the Quark Plasma. Phys. Rev., D36:2828, 1987.

[9] P. de Forcrand et al. Meson correlators in finite temperature lattice QCD. Phys. Rev., D63:054501, 2001.

[10] F. Karsch et al., Signatures of charmonium modification in spatial correlation functions. Phys. Rev., D85:114501, 2012.

[11] G. Boyd et al., Spatial and temporal hadron correlators below and above the chiral phase transition. Z. Phys., C64:331-338, 1994.

[12] I. Pushkina et al. Properties of hadron screening masses at finite baryonic density. Phys. Lett., B609:265-270, 2005.

[13] M. Cheng et al., Meson screening masses from lattice QCD with two light and the strange quark. Eur. Phys. J., C71:1564, 2011.

[14] E. Laermann and F. Pucci, Taste symmetry breaking at finite temperature. Eur. Phys. J., C72:2200, 2012.

[15] H. Iida et al., Hadron properties at finite temperature and density with two-flavor Wilson fermions. PoS, LATTICE2010:189, 2010.

[16] A. Bazavov et al., The chiral and deconfinement aspects of the QCD transition. Phys. Rev., D85:054503, 2012.

[17] A. Bazavov et al, Equation of state in ( 2+1 )-flavor QCD. Phys. Rev., D90:094503, 2014.

[18] G. Kilcup and S. Sharpe, A Tool Kit for Staggered Fermions. Nucl. Phys., B283:493-550, 1987.

[19] L. Levkova and C. DeTar. Charm annihilation effects on the hyperfine splitting in charmonium. Phys. Rev., D83:074504, 2011.

[20] G.P. Lepage et al., Constrained curve fitting. Nucl. Phys. Proc. Suppl., 106:12-20, 2002.

[21] F. Karsch et al., in preparation.

[22] V. Dick et al.,Microscopic origin of $U_{A}(1)$ symmetry violation in the high temperature phase of QCD. Phys. Rev., D91(9):094504, 2015.

[23] A. Bazavov et al, Strangeness at high temperatures: from hadrons to quarks. Phys. Rev. Lett., 111:082301, 2013.

[24] A. Bazavov et al, The melting and abundance of open charm hadrons. Phys. Lett., B737:210-215, 2014. 